\title{
Article \\ Machine Learning Reduced Gene/Non-Coding RNA Features That Classify Schizophrenia Patients Accurately and Highlight Insightful Gene Clusters
}

\author{
Yichuan Liu ${ }^{1}{ }^{\circledR}$, Hui-Qi Qu ${ }^{1} \mathbb{1}$, Xiao Chang ${ }^{1}$, Lifeng Tian ${ }^{1}{ }^{\circledR}$, Jingchun $Q u^{1}{ }^{1}$, Joseph Glessner ${ }^{1}$, \\ Patrick M. A. Sleiman ${ }^{1,2}$ and Hakon Hakonarson $1,2,3, *$ (D) \\ 1 Center for Applied Genomics, Children's Hospital of Philadelphia, Philadelphia, PA 19104, USA; \\ liuy5@email.chop.edu (Y.L.); quh@email.chop.edu (H.-Q.Q.); changx@email.chop.edu (X.C.); \\ tianl@email.chop.edu (L.T.); jingchun.qu789@gmail.com (J.Q.); glessner@email.chop.edu (J.G.); \\ sleimanp@email.chop.edu (P.M.A.S.) \\ 2 Division of Human Genetics, Department of Pediatrics, The Perelman School of Medicine, University of \\ Pennsylvania, Philadelphia, PA 19104, USA \\ 3 Department of Human Genetics, Children's Hospital of Philadelphia, Philadelphia, PA 19104, USA \\ * Correspondence: hakonarson@email.chop.edu; Tel.: +267-426-0088
}

\section{check for}

updates

Citation: Liu, Y.; Qu, H.-Q.; Chang,

X.; Tian, L.; Qu, J.; Glessner, J.;

Sleiman, P.M.A.; Hakonarson, H.

Machine Learning Reduced

Gene/Non-Coding RNA Features

That Classify Schizophrenia Patients Accurately and Highlight Insightful Gene Clusters. Int. J. Mol. Sci. 2021,

22, 3364. https://doi.org/10.3390/ ijms22073364

Academic Editor: Eric Vallender

Received: 3 March 2021

Accepted: 23 March 2021

Published: 25 March 2021

Publisher's Note: MDPI stays neutral with regard to jurisdictional claims in published maps and institutional affiliations.

Copyright: (c) 2021 by the authors. Licensee MDPI, Basel, Switzerland. This article is an open access article distributed under the terms and conditions of the Creative Commons Attribution (CC BY) license (https:/ / creativecommons.org/licenses/by/ $4.0 /)$.

\begin{abstract}
RNA-seq has been a powerful method to detect the differentially expressed genes/long non-coding RNAs (lncRNAs) in schizophrenia (SCZ) patients; however, due to overfitting problems differentially expressed targets (DETs) cannot be used properly as biomarkers. This study used machine learning to reduce gene/non-coding RNA features. Dorsolateral prefrontal cortex (dlpfc) RNA-seq data from 254 individuals was obtained from the CommonMind consortium. The average predictive accuracy for SCZ patients was $67 \%$ based on coding genes, and $96 \%$ based on long noncoding RNAs (lncRNAs). Machine learning is a powerful algorithm to reduce functional biomarkers in SCZ patients. The lncRNAs capture the characteristics of SCZ tissue more accurately than mRNA as the former regulate every level of gene expression, not limited to mRNA levels.
\end{abstract}

Keywords: schizophrenia; machine learning; transcriptome; long non-coding RNAs

\section{Introduction}

Schizophrenia (SCZ) is a complex biological disorder that involves combined effect of many genes, each conferring a small increase in susceptibility to the illness [1]. The redundancy of the gene networks underlying SCZ indicates that many gene combinations have the potential to result in a brain dysfunction that can manifest as SCZ or a related neurodevelopmental disorder [2]. Next-generation sequencing (NGS) enables measures of the transcriptome gene expression through RNA-seq, however, expressed genes cannot be used as biomarkers in many diseases that involve complex genetic networks, due to high noise level from a large number of genes and small number of samples. While the current solution is considering only differentially expressed targets (DETs) between SCZ and healthy controls, this often has multiple potential problems. For example, single or a small number of differentially expressed genes may not be clinically important for SCZ [3,4], indicating that a more comprehensive analysis is necessary to reveal the underlying genetic network for SCZ. Selection criteria for DETs are arbitrary, and while many researchers use adjusted $p$ value of 0.05 as a cut-off, this static standard often brings more ambiguity and neglects downstream analysis [5]. Even if only DETs were selected with a $p$ value cut-off as features for labeling or prediction, the DET number could still be too large which means that an overfitting problem could exist theoretically [6]. The key to address overfitting from too many features is to effectively reduce the number of features.

Beside coding genes, non-coding RNAs, especially long non-coding RNAs (lncRNAs) are important factors in shaping SCZ networks and are dynamically regulated by neuronal 
activation [7-10], and should therefore also be considered as potential feature vectors for SCZ gene network regulations. In this study, we acquired dorsolateral prefrontal cortex (dlpfc) samples' RNA-seq data from 254 subjects from the CommonMind consortium [11] (120 SCZ patients and 130 healthy controls, all non-Hispanic Caucasian). We then applied machine learning algorithms, including random forest, forward feature selection (ffs), and factor analysis to reduce the number of expressed genes into small list of feature vectors, in order to solve the overfitting problem. Two-fold shuffle tests showed that these selected feature vectors could accurately label SCZ patients versus controls. Selected genes were further clustered into gene modules through factor analysis, to explore potential functional units within the complex underlying genetic networks in SCZ.

\section{Results}

The statistical analysis and fold changes of genes were calculated. Altogether, 10,100 genes showed nominal significance with $p<0.05$ uncorrected for multiple testing (Table S1. Among the 10,100, expression of 3483 genes were down-regulated, and expression of 6617 genes were up-regulated. Using the WebGestalt (WEB-based Gene SeT AnaLysis Toolkit) web tool [12], over-representation analysis (ORA) by the Reactome approach [13] highlighted genes involved in mitochondrial function as down-regulated; and genes involved in gene transcription as upregulated (Tables S3 and S4).

\subsection{Accuracy Measure for Labeling Schizophrenia (SCZ) Patients Based on 2-Fold Shuffle Testing}

As described in the method section, 2-fold shuffle testing was applied to test the labeling prediction 50 times. Reduced genes, based on multiple machine learning methods, ranged from 36 to 282 , showed certain level of accuracy $(\sim 67 \%)$ in classifying SCZ patient's dorsolateral prefrontal cortex (dlpfc) samples versus healthy controls (Figure 1a). In contrast, to acquire a similar accuracy, we needed at least 100 top expressed coding genes to do random forest classification, whereas the accuracy of the traditional K-means clustering based on the top differentially expressed coding genes was only $50.2 \%$ at best. K-means clustering is an unsupervised classification method without requiring an independent training dataset. It is the simplest and the most popular method when the number of clusters was known. But still overfitting is possible as we identified the differentially expressed genes in the same dataset as features used for K-means, which means that the accuracy of $50.2 \%$ by K-means is possibly over-estimated. The actual performance of $\mathrm{K}$-means could be even worse than that of random forest. Machine learning methods reduced long non-coding RNAs (lncRNAs), ranging from 32 to 110 lncRNAs, and showing extremely high accuracy in classifying SCZ patients (accuracy level 99\%) (Figure 1b). In contrast, the accuracy of random forest classification based on the top differentially expressed lncRNAs was only $\sim 64 \%$, whereas the accuracy of the traditional K-means clustering based on the top differentially expressed lncRNAs was poor with the highest accuracy of $36.7 \%$. These machine learning results, demonstrating high accuracy indicate the existence of an functionally essential regulation network for SCZ brain tissues. The key difference between the clustering methods is the feature vector selections, in other words, the selection of genes/lncRNAs representing the essential differences between two groups (SCZ vs. controls). K-mean clustering used the 100 top differentially expressed genes, which is the most routine method, instead of multiple filtering steps of our machine learning methods. Considering gene co-expression network, overlapped information from the 100 top differentially expressed genes is always a concern. More importantly, the nature and complexity of SCZ have determined that the disease is not impacted by single or several genes, and the most differentially expressed genes are not necessarily the essential regulators. For lncRNAs, the issue is even enlarged because lncRNAs work as regulators of networks instead of expressed genes. 
(a)

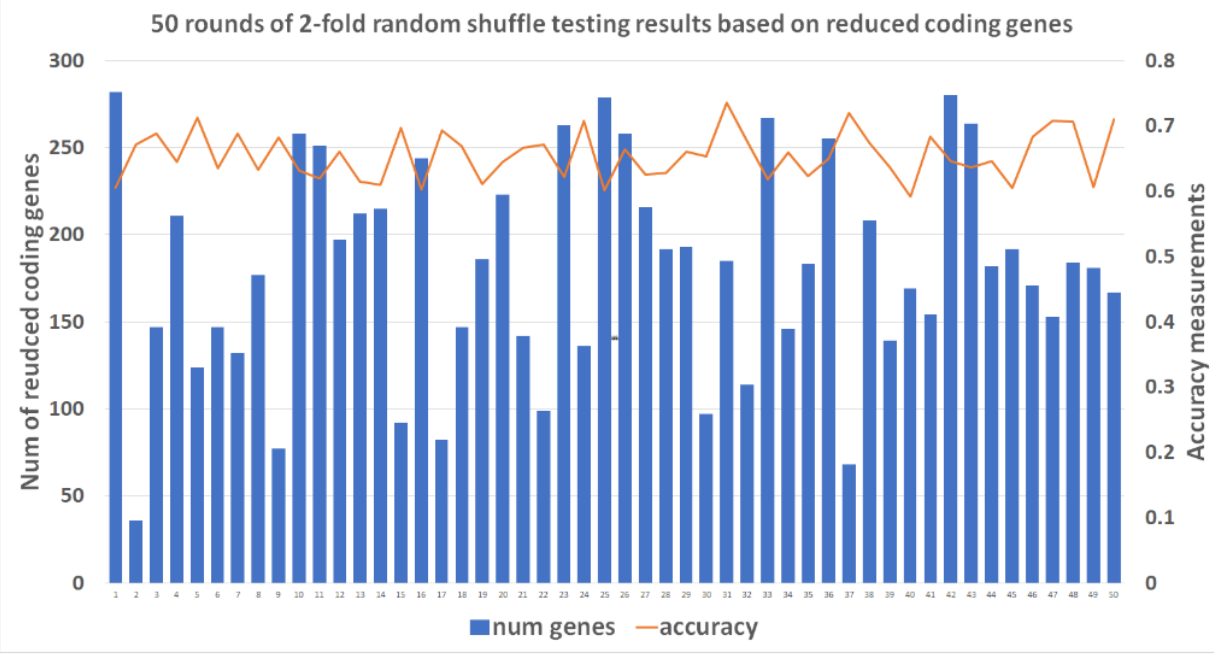

(b)

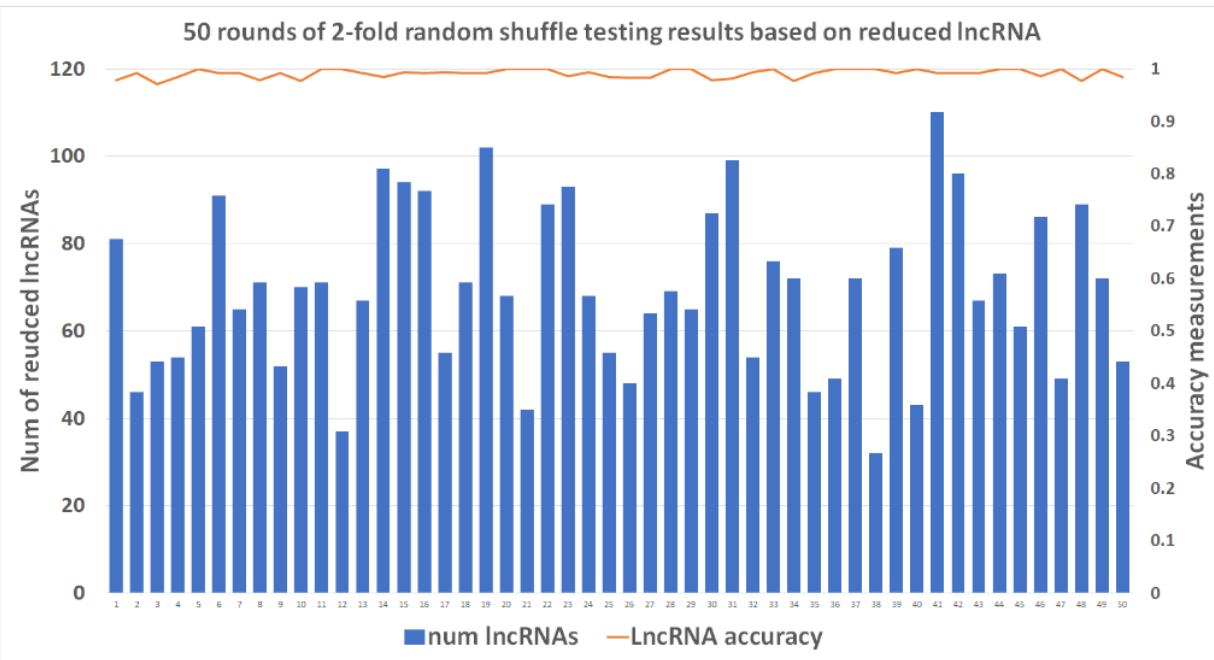

Figure 1. Two-fold random shuffle testing results for 50 rounds. $X$-axis is the round number, $Y_{-} 1$ axis (left) is the number of reduced genes (a) and lncRNAs (b), Y_2 axis (right) is the accuracy measurement ranged from 0 to 1 .

\subsection{Selected Gene/lncRNA Feature Based on Machine Learning Algorithm}

After multiple layers of filtering, including the machine learning methods, the number of genes reduced from 27,101 to 734 in the total of 254 dorsolateral prefrontal cortex (dlpfc) samples (Figure 2a). Expressions of all these genes have nominally statistical significance with $p$ value range from $1.5 \times 10^{-9}$ to $6.45 \times 10^{-3}$. Among the 734 genes (Table S5), 412 were downregulated; and 322 genes were upregulated. These genes were found to be enriched in glycosaminoglycan metabolic processes (adjusted $p$ value $=3.1 \times 10^{-3}$ ), aminoglycan metabolic processes (adjusted $p$ value $=3.7 \times 10^{-3}$ ), mucopolysaccharide metabolic processes (adjusted $p$ value $=1.2 \times 10^{-2}$ ), based on Gene Ontology [14]. A total of 13,871 long non-coding RNAs (lncRNAs) identified in GENCODE were reduced to 605 using comparable pipelines. The results suggest that by combining multiple machine learning methods is a powerful tool to reduce the number of gene features which represent the variations of the data. It is also worth mentioning that only 255 of the 734 coding genes have differential expression with False Discovery Rate (FDR) Adjusted $p$ value $<0.05$. 
(a)

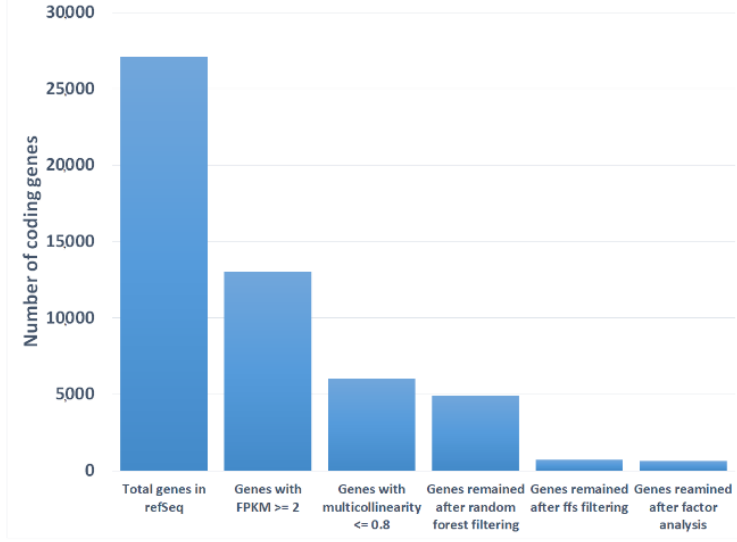

(c)

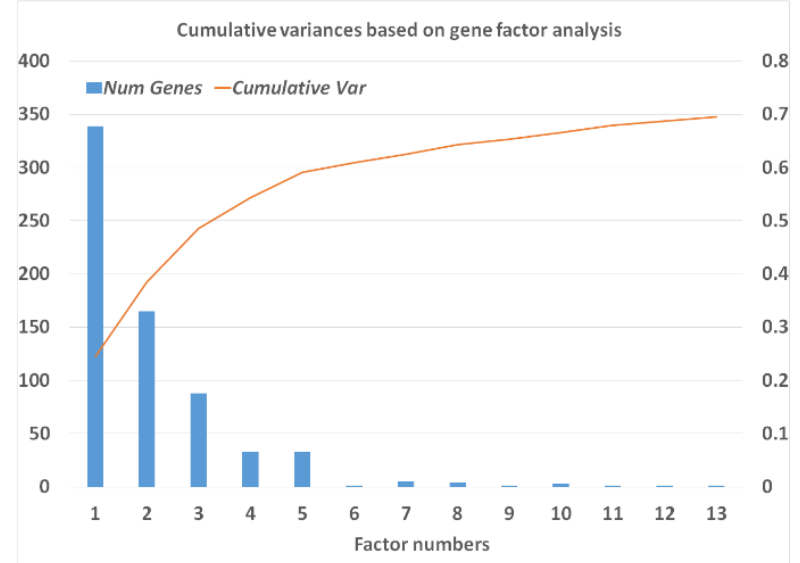

(b)

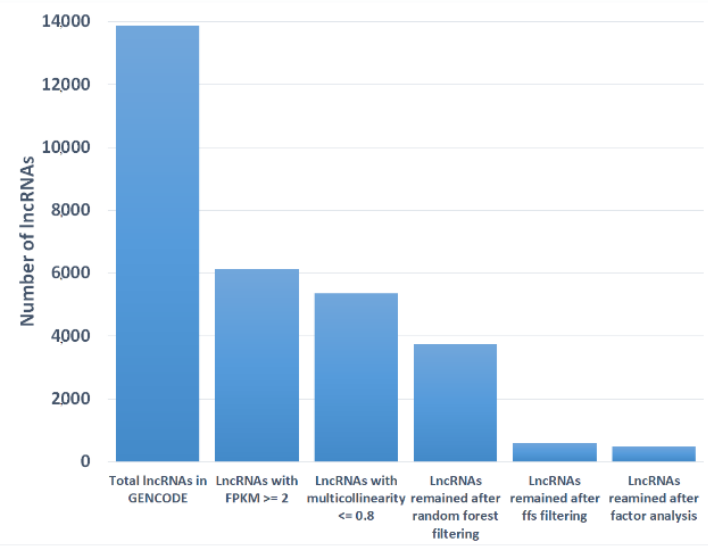

(d)

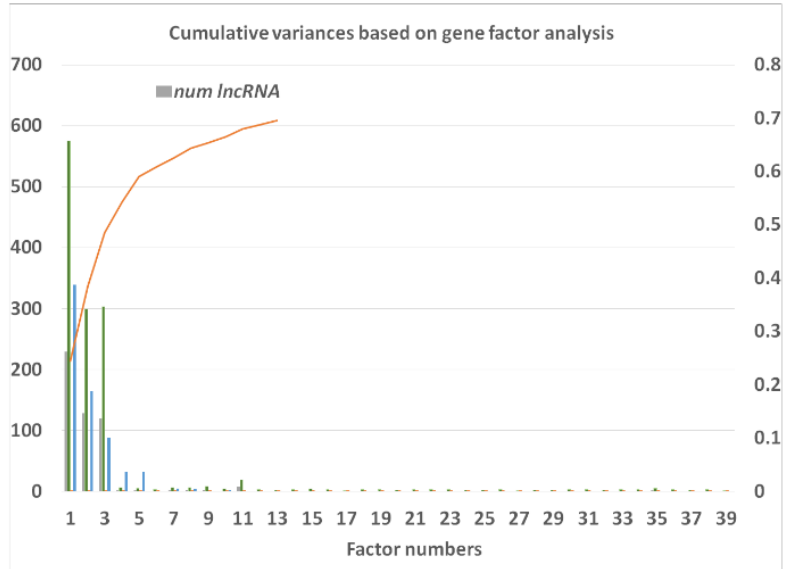

Figure 2. (a) Number of feature vectors for coding genes after multiple filtering methods; (b) number of feature vectors for lncRNAs after multiple filtering methods; (c) factor analysis cumulative curve and number of remain coding-gene feature vectors; (d) factor analysis cumulative curve and number of remain lncRNAs and their targeted genes' feature vectors.

\subsection{Factors and Potential Gene Modules from Factor Analysis}

The purpose of factor analysis is to group the reduced feature vectors genes/lncRNAs into modules, which could function together for a complex disease as SCZ. The 734 genes were clustered into 66 factors, where the first 13 factors contribute $~ 70 \%$ of the variances (Figure 2c, Table S5). Factor 1 contains 339 genes and contributes $24.5 \%$ of the total variance, all genes in factor 1 are down regulated in SCZ versus health controls, as shown by differential expression in CommonMind consortium database [15]. Not unexpectedly, the over-representation analysis (ORA) by Human Phenotype Ontology (HPO) shows that factor 1 , as the major factor of SCZ transcriptome, contains a number of genes critical in neurodevelopment that are involved in various brain disorders, including HP:0001298_Encephalopathy, HP:0001098_Abnormal fundus morphology, and HP:0004329_Abnormal morphology of the posterior segment of the globe. Enrichment analysis by biological pathways using the expressed gene list (FPKM $\geq 2$ ) as the background (Figure 3a) highlighted sulfur compound biosynthetic processes (FDR adjusted $p$ value $=4.8 \times 10^{-4}$ ), glycosaminoglycan metabolic processes (FDR adjusted $p$ value $=8.1 \times 10^{-3}$ ), and glycoprotein metabolic processes (FDR adjusted $p$ value $=0.019$ ). Eight genes were identified in previous PG2 GWAS studies [16], including APH1A (rs140505938, FDR adjusted $p$ value $=4.49 \times 10^{-10}$ ), ASPHD1 (rs11646127, FDR adjusted $p$ value $\left.=4.55 \times 10^{-11}\right), B R I N P 2($ rs6670165, FDR ad justed $p$ value $\left.=4.45 \times 10^{-8}\right)$, CHRM4 (rs7951870, FDR adjusted $p$ value $\left.=1.26 \times 10^{-11}\right)$, INO80E (rs11646127, FDR adjusted $p$ value $\left.=4.55 \times 10^{-11}\right)$, PCCB $($ rs7432375, FDR adjusted 
$p$ value $\left.=7.26 \times 10^{-11}\right), \operatorname{SPCS1}\left(\right.$ rs3617, FDR adjusted $p$ value $\left.=4.26 \times 10^{-11}\right)$, and TAC3 (rs61937595, FDR adjusted $p$ value $=2.02 \times 10^{-12}$ ), all these hotspots were also identified in CLOZUK, which SCZ cases were ascertained with the assistance of Novartis, and the samples consisted of individuals with treatment-resistant schizophrenia according to the clozapine registration forms completed by treating psychiatrists [17]. Of note, 159 out of the 339 genes $(46.9 \%)$ have at least one supportive evidence from previous knowledge, and 12 genes have at least four supportive evidences (Table 1 ).

[a]

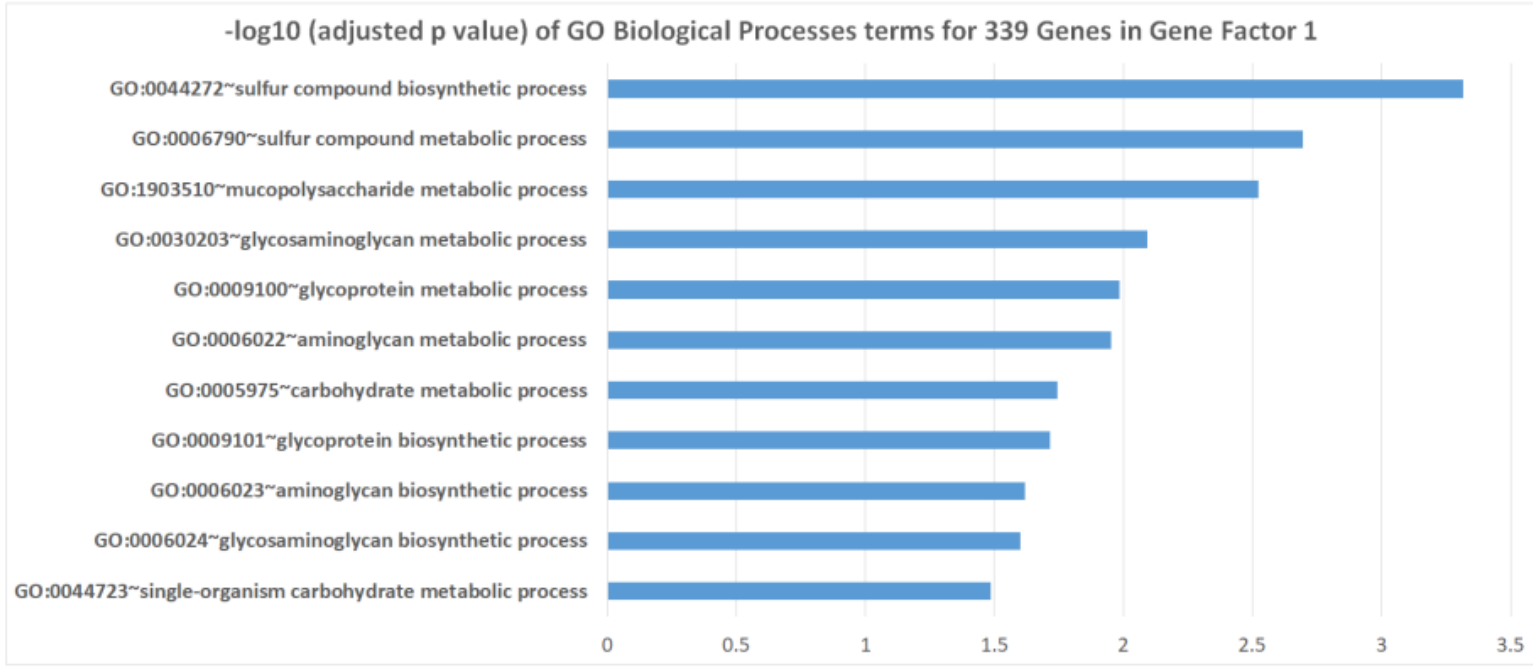

[b]

$-\log 10$ (adjusted p value) of GO functional enriched terms for 321 targeted Genes in IncRNA factor 2

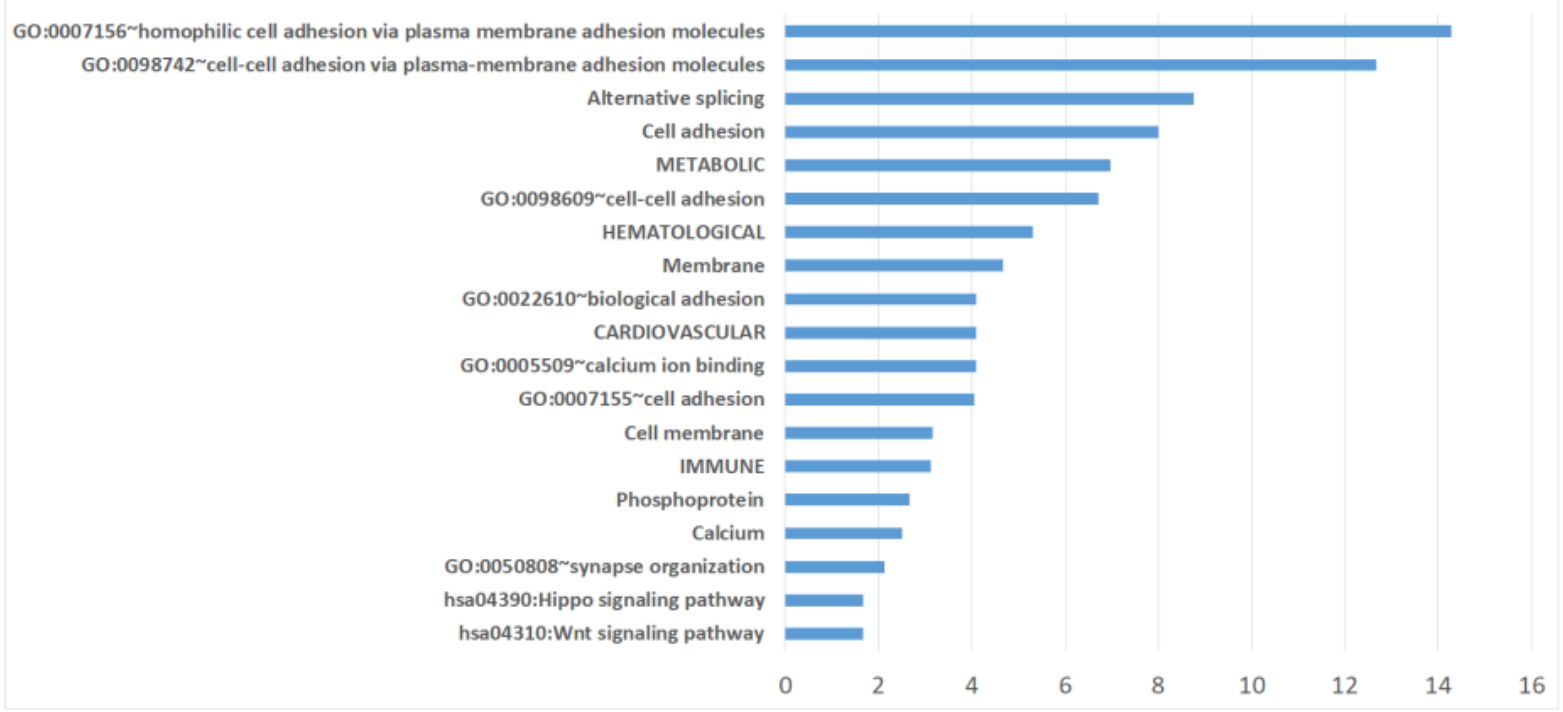

Figure 3. $-\log 10$ (Adjusted $p$ value) scale for enriched functional pathways: (a) gene factor 1; (b) targeted genes in lncRNA factor 2 .

A total of 166 genes clustered into factor 2 and majority of them (94\%) are up regulated in SCZ. Factor 2 contributes 13.9\% of the data variance (Figure 2c, Table S6), 11 genes $(6.7 \%)$ were found to be differentially expressed and none of them are down regulated in SCZ. A total of 65 out of 165 genes (39.3\%) have at least one supportive evidence from previous knowledge, and 5 genes have at least four supportive evidences (Table 1). Enrichment analysis by biological pathways highlighted phosphorylation (FDR adjusted 
$p$ value $=5.1 \times 10^{-3}$ ). There are 88,33 , and 33 genes in factor 3,4 and 5 (Table S6), respectively, contributing $10 \%, 5.7 \%$ and $4.8 \%$ of the variance, respectively.

Table 1. Genes in gene factors with high supportive evidences, including genome-wide association study (GWAS), Genome Wide Linkage Study (Linkage) Copy Number Variation (CNV), integrative analysis (Integrative), differentially methylated (Diff Methy), Differentially expressed (Diff Exp), identified by exome sequencing (Exome), expression level in brain tissues (Brain Exp), Gene Ontology (GO), and total score (Score)

\begin{tabular}{|c|c|c|c|c|c|c|c|c|c|c|c|}
\hline Factor & Gene & GWAS & Linkage & $\mathrm{CNV}$ & Integrative & Diff Methy & Diff Exp & Exome & Brain Exp & GO & Score \\
\hline 1 & ASPHD1 & 1 & 0 & 1 & 0 & 0 & 1 & 1 & $1(26.68)$ & 0 & 5 \\
\hline 1 & AK4 & 0 & 0 & 0 & 0 & 1 & 1 & 0 & $1(13.55)$ & 1 & 4 \\
\hline 1 & APH1A & 1 & 0 & 0 & 0 & 0 & 0 & 1 & $1(43.24)$ & 1 & 4 \\
\hline 1 & FPGS & 0 & 0 & 0 & 0 & 1 & 1 & 0 & $1(14.38)$ & 1 & 4 \\
\hline 1 & FSCN1 & 0 & 0 & 0 & 0 & 1 & 1 & 1 & $1(48.13)$ & 0 & 4 \\
\hline 1 & INO80E & 0 & 0 & 1 & 1 & 1 & 0 & 0 & $1(20.22)$ & 0 & 4 \\
\hline 1 & P2RX6 & 0 & 0 & 1 & 0 & 1 & 1 & 0 & $0(4.99)$ & 1 & 4 \\
\hline 1 & PCCB & 1 & 0 & 0 & 1 & 1 & 0 & 0 & $1(26.64)$ & 0 & 4 \\
\hline 1 & PRODH & 0 & 1 & 0 & 0 & 0 & 1 & 1 & $1(24.04)$ & 0 & 4 \\
\hline 1 & SCN1B & 0 & 0 & 0 & 0 & 1 & 1 & 0 & $1(25.12)$ & 1 & 4 \\
\hline 1 & SEMA7A & 0 & 0 & 0 & 0 & 1 & 1 & 0 & $1(13.64)$ & 1 & 4 \\
\hline 2 & BCCIP & 0 & 0 & 0 & 0 & 1 & 1 & 1 & $1(26.92)$ & 1 & 5 \\
\hline 2 & HNRNPU & 0 & 0 & 0 & 0 & 1 & 1 & 1 & $1(108.84)$ & 0 & 4 \\
\hline 2 & HSP90AA1 & 0 & 0 & 0 & 0 & 0 & 1 & 1 & $1(411.41)$ & 1 & 4 \\
\hline 2 & NRG1 & 0 & 1 & 0 & 0 & 1 & 0 & 1 & $0(4.47)$ & 1 & 4 \\
\hline 2 & PDE4B & 1 & 1 & 0 & 0 & 0 & 0 & 0 & $1(38.88)$ & 1 & 4 \\
\hline 3 & TIMP2 & 0 & 0 & 0 & 0 & 0 & 1 & 1 & 1 (107.98) & 1 & 4 \\
\hline 7 & BCL6 & 0 & 0 & 0 & 0 & 1 & 1 & 1 & $1(29.50)$ & 1 & 5 \\
\hline 9 & RERE & 1 & 0 & 0 & 1 & 0 & 0 & 0 & $1(25.51)$ & 1 & 4 \\
\hline
\end{tabular}

For lncRNAs, factor analysis resulted in 91 factors, where the first 45 factors (contain 496 lncRNAs) explain $~ 70 \%$ of the variations of the expression data (Figure 2d, Table S7). The first 3 factors of lncRNA factor analysis explained $21 \%, 14 \%$, and $13 \%$ variance respectively, with a subtotal of $48 \%$. Among the 496 lncRNAs, only 88 have differential expression with FDR Adjusted $p$ value $<0.05$. The genes closest to the lncRNA binding sites were identified as their target genes. Mapping lncRNAs and their targets is a challenging problem. Computational methods cannot effectively solve the false positive issue, and literature-based databases usually have low sensitivity. Previous studies [18-20] showed that lncRNAs tend to regulate expression of neighboring protein-coding genes and thus contribute to the regulation of mRNA and protein levels in mammal. Therefore, choosing the closest genes of the lncRNA could capture at least a certain level of corresponding target genes and be more practical in the application. However, due to gene functional overlapping, choosing only the closest genes may indeed introduce bias. In the trade-off between high sensitivity and acceptable specificity, based on our results and current literature, three closest genes is plausibly the most reasonable choice of the selection.

A total of 1193 genes were selected and found to be enriched in multiple disease category [21], METABOLIC (FDR adjusted $p$ value $\left.=2.8 \times 10^{-5}\right)$, CARDIOVASCULAR $($ FDR adjusted $p$ value $=3.2 \times 10^{-2}$ ), HEMATOLOGICAL (FDR adjusted $p$ value $=7.3 \times 10^{-3}$ ), IMMUNE (FDR adjusted $p$ value $=4.8 \times 10^{-2}$ ), and post translational modifications such as phosphorylation $\left(8.2 \times 10^{-4}\right)$. Multiple neurodevelopmental related pathways which cannot be captured by the mRNA pathway analysis, are highlighted for the lncRNAs' targeted gene sets, including regulation of transferase activity (FDR adjusted $p$ value $=1.8 \times 10^{-3}$ ), neuron projection morphogenesis (FDR adjusted $p$ value $=4.8 \times 10^{-3}$ ), positive regulation of nervous system development (FDR adjusted $p$ value $=4.6 \times 10^{-3}$ ); regulation of kinase activity (FDR adjusted $p$ value $=5.7 \times 10^{-3}$ ), neuron differentiation (FDR adjusted $p$ value $\left.=8.3 \times 10^{-3}\right)$, and neuron projection development (FDR adjusted 
$p$ value $\left.=8.8 \times 10^{-3}\right)$. Target genes in lncRNA factors with high supportive evidence are shown in Table 2.

Table 2. Target genes in lncRNA factors with high supportive evidence.

\begin{tabular}{|c|c|c|c|c|c|c|c|c|c|c|c|c|}
\hline Factor & lncRNA & $\begin{array}{l}\text { Target } \\
\text { Gene }\end{array}$ & GWAS & Linkage & $\mathrm{CNV}$ & Integrative & $\begin{array}{c}\text { Diff } \\
\text { Methy }\end{array}$ & $\begin{array}{l}\text { Diff } \\
\text { Exp }\end{array}$ & Exome & Brain Exp & GO & Score \\
\hline 1 & ENSG00000247735.2 & ASPHD1 & 1 & 0 & 1 & 0 & 0 & 1 & 1 & $1(26.68)$ & 0 & 5 \\
\hline 1 & ENSG00000232912.1 & RERE & 1 & 0 & 0 & 1 & 0 & 0 & 0 & $1(25.51)$ & 1 & 4 \\
\hline 1 & ENSG00000235770.1 & FN1 & 0 & 1 & 0 & 0 & 0 & 0 & 1 & $1(38.33)$ & 1 & 4 \\
\hline 1 & ENSG00000235831.2 & ITPR1 & 0 & 0 & 0 & 0 & 1 & 0 & 1 & $1(25.59)$ & 1 & 4 \\
\hline 1 & ENSG00000239569.2 & SRPK2 & 1 & 0 & 0 & 0 & 1 & 0 & 0 & $1(75.17)$ & 1 & 4 \\
\hline 1 & ENSG00000243762.1 & RANBP1 & 0 & 1 & 1 & 0 & 1 & 0 & 0 & $1(30.73)$ & 0 & 4 \\
\hline 1 & ENSG00000247735.2 & SEZ6L2 & 1 & 0 & 1 & 0 & 0 & 0 & 0 & $1(38.77)$ & 1 & 4 \\
\hline 1 & ENSG00000257126.1 & FOXG1 & 1 & 0 & 0 & 0 & 1 & 0 & 0 & $1(22.05)$ & 1 & 4 \\
\hline 1 & ENSG00000261220.2 & ST3GAL1 & 0 & 0 & 0 & 0 & 1 & 0 & 1 & $1(8.93)$ & 1 & 4 \\
\hline 1 & ENSG00000271849.1 & PJA2 & 0 & 0 & 0 & 0 & 1 & 0 & 1 & $1(144.54)$ & 1 & 4 \\
\hline 2 & ENSG00000224563.1 & BCL6 & 0 & 0 & 0 & 0 & 1 & 1 & 1 & $1(29.50)$ & 1 & 5 \\
\hline 2 & ENSG00000226978.1 & MAGI2 & 0 & 1 & 0 & 0 & 1 & 0 & 0 & $1(24.46)$ & 1 & 4 \\
\hline 2 & ENSG00000236031.1 & AKT3 & 1 & 0 & 0 & 0 & 0 & 0 & 1 & $1(35.93)$ & 1 & 4 \\
\hline 2 & ENSG00000248816.1 & TENM3 & 0 & 0 & 0 & 0 & 1 & 1 & 0 & $1(8.06)$ & 1 & 4 \\
\hline 2 & ENSG00000272367.1 & RASA1 & 0 & 0 & 0 & 0 & 1 & 1 & 0 & $1(20.45)$ & 1 & 4 \\
\hline 3 & ENSG00000272989.1 & DLG1 & 0 & 0 & 1 & 0 & 0 & 1 & 1 & $1(52.38)$ & 1 & 5 \\
\hline 3 & ENSG00000239569.2 & SRPK2 & 1 & 0 & 0 & 0 & 1 & 0 & 0 & $1(75.17)$ & 1 & 4 \\
\hline 3 & ENSG00000273164.1 & PRODH & 0 & 1 & 0 & 0 & 0 & 1 & 1 & $1(24.04)$ & 0 & 4 \\
\hline 3 & ENSG00000273164.1 & DGCR2 & 0 & 1 & 1 & 0 & 0 & 0 & 1 & $1(30.80)$ & 0 & 4 \\
\hline 37 & ENSG00000248816.1 & TENM3 & 0 & 0 & 0 & 0 & 1 & 1 & 0 & $1(8.06)$ & 1 & 4 \\
\hline
\end{tabular}

Consequently, we tested the machine learning algorithm in an independent dataset including 22 non-EA SCZ and 27 controls. The performance with coding genes was highly reproducible with the accuracy of $95 \%$, whereas the accuracy with lncRNAs was only $37 \%$.

\section{Discussion}

Growing evidence indicates that distinct neuronal ncRNAs, particularly lncRNAs, are likely to influence the development of neurodevelopmental diseases, including SCZ [7]. However, to date, neither genes in neurodevelopmental networks nor the lncRNAs in regulation processes have been successfully applied as feature vectors to label the phenotypic status of the patients. The main obstacle is the number of genes/lncRNAs as predictive features is huge (over 20,000 genes and 10,000 lncRNAs), and the number of biological samples, especially brain tissues, is usually small due to the difficulty in sample collections. Therefore, predictive models are deemed to fail due to overfitting issues. Currently, the solution for this problem is to select a small number of genes that are differentially expressed between SCZ and controls. While this may help, the algorithm and criteria used for selecting these gene remains controversial, and genes contributing to the network with small effects and less statistical significance may be missed.

Machine learning methods have been proven to be effective in reducing the feature vectors while capturing essential data differences in studies of many fields, include genetic expression studies [22,23]. In this study, we applied multiple machine learning layers for expressed genes in dorsolateral prefrontal cortex (dlpfc) RNA-seq data from 254 samples from the CommonMind consortium, in order to show that reduced gene/lncRNA features could accurately classify SCZ patients versus healthy controls. Combining machine learning methods, such as random forest and forward feature selection (ffs) for expressed genes/lncRNAs, the number of genes was significantly reduced from over 25,000 to an average $\sim 180$ genes while the IncRNA number was reduced from 13,000 to on average $\sim 70$ through the simulations. The 2-fold shuffle tests (samples split to 1:1 ratio, half used as training data and rests used as testing data) applied in 50 separate rounds (Figure 1) shows that reduced gene feature vector has modest power ( $\sim 67 \%$ accuracy) in classifying SCZ patients versus healthy controls, whereas lncRNAs could serve as an effective predictor 
( $\sim 99 \%$ accuracy). On the other hand, the random forest classification tends to require a larger number of top differentially expressed coding genes to get similar accuracy as that of machine learning selected genes, as top differentially expressed coding genes may not be independent of each other because of gene co-expression networks. The random forest classification based on top differentially expressed lncRNAs has poor performance, while with IncRNAs as regulators highly differential lncRNAs may not be the most important lncRNAs. These results demonstrate that machine learning has the potential to be an alternative methods to detect the essential differences of gene expressions in SCZ, also the regulation networks involved in IncRNAs are more stable than gene expression networks, in other words, gene expressions remain highly diverse for different persons but the lncRNAs expression seems universal among different individuals. More importantly, as demonstrated by our findings that the average predictive accuracy for SCZ patients is $67 \%$ based on coding genes, and $96 \%$ based on lncRNAs, lncRNAs represent a more accurate biomarker for the SCZ transcriptome. The lncRNAs regulates every level of gene expression, including but not limited to mRNA levels, which may explain why lncRNAs capture the characteristics of SCZ tissue more accurately [24]. Considering gene co-expression network, duplicated/overlapped information from top differentially expressed mRNAs is always a concern. On the other hand, too many mRNAs will cause overfitting unavoidably. Our study, therefore, suggests lncRNAs are more informative and better features.

Furthermore, the reduced gene/lncRNA features were clustered based on factor analysis to form the gene modules. Our study showed that major factors were enriched for genes important in neurodevelopment and brain disorders, thereby proving the validity of the dimension reduction process. While genes in major factors show enrichment of SCZ related pathways or neurodevelopmental associated network and can serve as a proof-ofprinciple of this study, other factors may harbor novel knowledge about SCZ and warrant further study. Genes within each factor have higher portion of SCZ supportive evidences while known differentially expressed genes only counted small portion of genes in each factor (Figure 4). Combining these clues together indicates that the machine learning models capture contributing genes more effectively compared to traditional differential expression tests. SCZ genetics involves combined effect of many genes, each conferring a small increase in susceptibility to the illness [25].

Genes in certain factors highlighted SCZ-associated networks and the biochemical molecules, synthesis/metabolism, as neuro system modulators. For example, 339 genes in gene factor 1 were enriched in sulfur compound biosynthetic and metabolic processes (adjusted $p$ value $=4.8 \times 10^{-3}$ ). Sulfur is an essential chemical for proteinogenic amino acid methionine (Met), and methionine-folate cycle-dependent one-carbon metabolism is implicated in the pathophysiology of SCZ while deficiencies in the one-carbon metabolism components folate is consistent findings in SCZ patients [26]. These genes are also enriched in glycosaminoglycan metabolic processes (adjusted $p$ value $=8.1 \times 10^{-3}$ ). Glycosaminoglycans, an alternative name of mucopolysaccharides, play critical roles in the normal function of the central nervous system [27]. Abnormal glycosaminoglycan synthesis in cerebral cortex have been reported to associated with SCZ [28]. Hyaluronic acid, one of the major classes of glycosaminoglycans, is a critical component on the surface of perineuronal nets (PNNs), while a decreased number of PNNs is associated with schizophrenia [29]. Other enriched chemical synthesis procedures for genes in factor 1 include glycoprotein metabolic processes (adjusted $p$ value $=0.019$ ) where $p$-glycoprotein a major efflux pump in the blood-brain barrier, has a profound effect on entry of drugs, peptides and other substances into the central nervous system [30]. For lncRNA factor 2, 321 targeted genes (the union of 128 lncRNAs' top three closest genes) were significantly enriched in cell adhesion $\left(\right.$ adjusted $p$ value $\left.=9.7 \times 10^{-9}\right)$, phosphorylation $\left(\right.$ adjusted $p$ value $\left.=2.2 \times 10^{-3}\right)$, SCZ associated pathways include calcium ion binding, while dysregulation of neural calcium has been found signaling in SCZ [31] (adjusted $p$ value $=8.1 \times 10^{-5}$ ) as well as the Wnt signaling pathway, a crucial pathway in neurodevelopment and in regulating the function and structure of the adult nervous system [32] (adjusted $p$ value $=0.022$ ) (Figure 3b). In 
contrast, many factors although with high consistent regulation tendency have no known genetic functions. For example, gene factor 2 (94\% genes up regulated in SCZ, 39.3\% have at least one supportive evidence, and only $11 \%$ of the genes are known to be differentially expressed in SCZ), gene factor 3 (78\% genes up regulated in SCZ, 31.8\% have at least one supportive evidence, and only $4.5 \%$ of the genes are known to be differentially expressed), gene factor 4 (70\% genes down regulated in SCZ, $42.4 \%$ have at least one supportive evidence, and only $15 \%$ of the genes are known to be differentially expressed), and lncRNA factor 1, which contains 229 lncRNAs (597 target genes) but no enrichment were identified for biological functions. Taken together, these factors are potential targets for researchers to explore in further studies. One of the limitations of this study is the selection of target genes. We chose 3 closest genes of a lncRNA to capture the corresponding target genes based on previous studies showing that lncRNAs tend to regulate the expression of neighboring protein-coding genes [18-20]. The mapping procedure of lncRNAs to their target genes is currently a challenging problem and without a gold standard. We chose the 3 closest genes of a lncRNA to balance the trade-off between high sensitivity and acceptable specificity. More sophisticated approaches are still warranted. In addition, we emphasize that, as an exploratory study with limitations, the prediction model created in this study warrants further validation in other datasets, especially in different ethnicities. As shown by our data in a small sample of non-EA individuals from the CommonMind database (including $22 \mathrm{SCZ}$ and 27 controls), the prediction accuracy of coding genes was reproducible, but not that of lncRNAs. In contrast to coding genes directly determining the pathophysiology of SCZ, lncRNAs have only regulatory and indirect effects in SCZ. Their prediction performance might thus be inferior if applied to a different ethnicity.

[a]

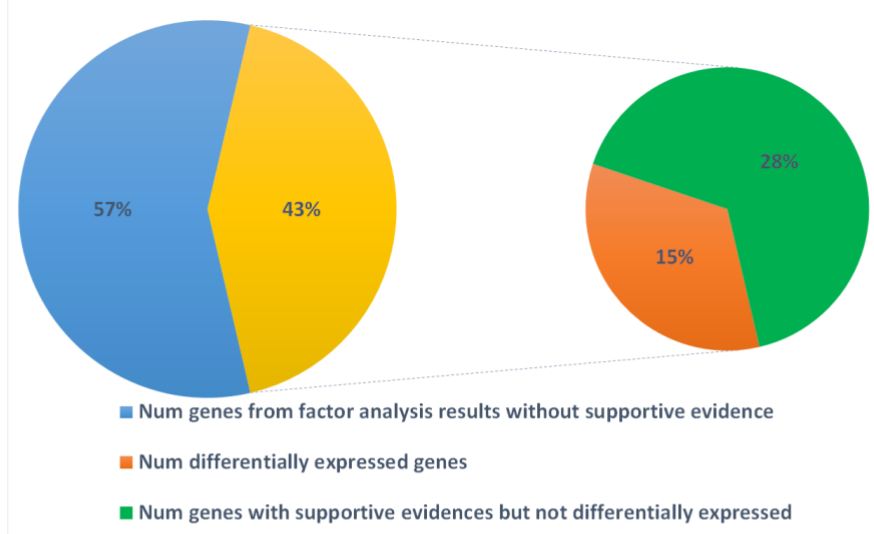

[b]

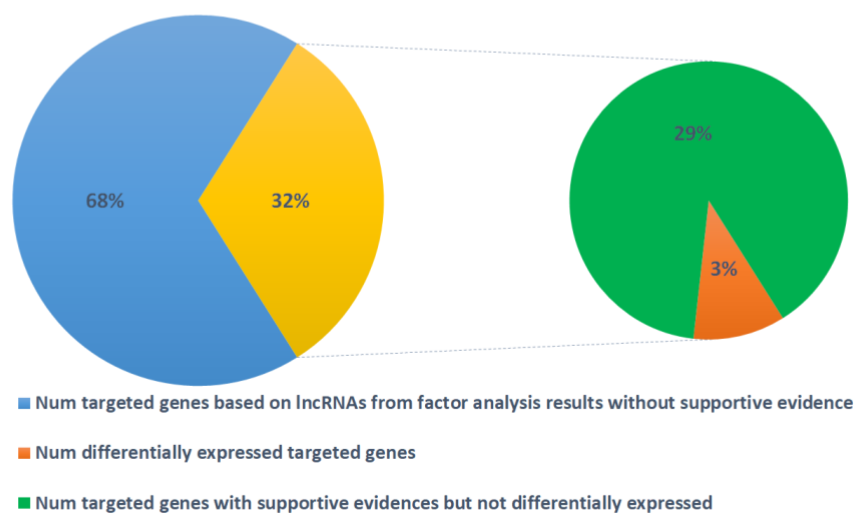

Figure 4. Portion of differentially expressed genes/lncRNAs versus genes/lncRNAs with at least one supportive evidence: (a) coding genes after factor analysis; (b) targeted coding genes based on lncRNA genomic locus. 


\section{Methods \& Materials}

\subsection{RNA-Seq Data for Dorsolateral Prefrontal Cortex (DLPFC) Samples}

This study was approved by The Children's Hospital of Philadelphia (CHOP) Institutional Review Board (IRB). All the patients who participated in this project were consented and they agree to the publication of the results. RNA-seq data of dorsolateral prefrontal cortex (DLPFC) samples were obtained from the CommonMind consortium [11]. More specifically, the RNA-seq data were downloaded from the CommonMind Consortium Knowledge Portal at synapse (https: / /www.synapse.org/\#!Synapse:syn2759792/wiki/69613) accessed on 1 January 2017.

To minimize the confounding effect of ethnicity, we only selected SCZ patients and controls who are of European ancestry (EA). A total of 254 RNA-seq BAM files were obtained include 120 SCZ patients and 134 healthy controls. Samples with a minimum of 50 million mapped reads and less than 5\% rRNA-aligned reads were retained for downstream analysis. The RNA-seq data were aligned using the Spliced Transcripts Alignment to a Reference (STAR) [33]. Details for individuals, such as gender, age, and read counts/unique reads were listed in Table S2.

Based on the consortium's description, RNA was isolated from $50 \mathrm{mg}$ homogenized tissue in Trizol using the RNeasy kit based on the instructional protocol. The mean total RNA yield was $15.3 \mu \mathrm{g}( \pm 5.7)$. The RNA integrity number (RIN) was determined by fractionating RNA samples on the 6000 Nano chip (Agilent Technologies, Santa Clara, CA, USA) on the Agilent 2100 Bioanalyzer. The mean RIN was $7.7( \pm 0.9)$, and the mean ratio of $260 / 280$ was $2.0( \pm 0.02)$. Processing order was re-randomized prior to ribosomal RNA (rRNA) depletion. Briefly, rRNA was depleted from about $1 \mu \mathrm{g}$ of total RNA using a Ribo-Zero Magnetic Gold kit (Illumina/Epicenter Cat \# MRZG12324) to enrich for polyadenylated coding RNA and non-coding RNA. The sequencing library was prepared using the TruSeq RNA Sample Preparation Kit v2 (RS-122-2001-48 reactions) in batches of 24 samples. A pool of 10 barcoded libraries was layered on a random selection of two of the eight lanes of the Illumina flow cell bridge amplified to 250 million raw clusters. One-hundred base pair paired end reads were obtained on a HiSeq 2500. The sequence data were processed for primary analysis to generate Quality Control (QC)values.

\subsection{Gene/Non-Coding RNAs Expression Matrix}

The genomic template used for coding genes expressions is hg19 refSeq, and long noncoding RNAs template is GENCODE version 19 [34]. The expression matrix was generated based on Cuffnorm functions in Cufflink package version 2.2.1 [35], and the SCZ and controls groups are normalized. More specifically, the Cuffnorm reports expression values in Fragments Per Kilobase (FPKM) for each gene were properly normalized based on library size. To eliminate potential noisy signals, the gene expression FPKM values less than 2 were removed due to potential noises from low FPKM genes. Genes/lncRNAs with collinearity over $80 \%$ were removed because generally feature selection methods assume feature vectors are independent to each other. Differential expression analysis was undertaken by independent Student's $t$-tests based on the FPKMs after the classic-FPKM normalization.

\subsection{Gene Reductions Using Machine Learning Algorithms}

Multiple machine learning algorithms include random forest, forward feature selection (ffs), and factor analysis, were applied to select and reducing the informative gene/lncRNA features between SCZ and controls. Random forest is one of the most widely used algorithms for feature selection, which computes relative importance or contribution of each gene feature in the prediction, then scales the relevance down so that the sum of all scores is 1 . All the genes/lncRNAs with zero relative importance were removed. The following parameters were applied for the random forest model, including: the function to measure the quality of a split, using "gini"; the minimum number of samples required to split an internal node equals 2; and nodes are expanded until all leaves are pure or until all 
leaves contain less than 2 . The number of features to consider when looking for the best split equals the square root (num_features) and the number of trees in the forest equals 500 .

The second algorithm forward feature selection (ffs) is one of the most common methods to reduce number of features for machine learning inputs by trying to find the best features which improve the performance of the model. The modeling codes are based on based on the Scikit-learn package (version 0.21.3) in Python language [36].

In order to test the predictive abilities for selected gene/lncRNA features, we applied a 2-fold shuffle testing. In other words, the SCZ and control samples were split into 1:1 ratio for 50 rounds randomly, one set used as training data and another one used as independent testing set. Gene/lncRNA features were selected as described in previous paragraph for training data (to overcome overfitting problem, the only parameter altered is we required random forest relative importance $>0.0005$ rather than $>0$ ), then a random forest classifier is applied to label whether the sample is SCZ or control in testing data based on training data gene/lncRNA features.

Factor analysis was applied to the entire sample set for further clustering gene/lncRNA features. Factor analysis is a statistical method used to describe variability among observed, correlated variables in terms of a potentially lower number of unobserved variables called factors, and the methods have been proven to be a good interpreter for gene networks and pathways [37,38]. The number of factors chosen in the model was 50 for both coding genes and lncRNAs based on Kaiser-Meyer-Olkin (KMO) test (when eigenvalues are greater than one), and the rotation method, "varimax". The Python-based factor_analyzer package was used in the analysis (version 0.3.1).

For validation, we further tested the machine learning algorithm in an independent dataset, i.e., a small sample of non-EA individuals available from the CommonMind database (including $22 \mathrm{SCZ}$ and 27 controls).

Supportive evidences for SCZ genes were collected based on SZDB, a database contains various layers of data of schizophrenia researches, such as genetic data, copy number variants (CNVs) data, whole genome/exome sequencing (WGS/WES) data, gene expression data, functional genomics data, and protein-protein interaction data [39].

Supplementary Materials: The following are available online at https: / www.mdpi.com/1422-0 067/22/7/3364/s1, Table S1. The statistical analysis and fold changes of gene expression; Table S2. Details of the research subjects; Table S3. Downregulated genes pathways; Table S4. Upregulated genes pathways; Table S5. Selected Gene features based on Machine learning algorithm; Table S6. Factors of coding genes; Table S7. lncRNAs.

Author Contributions: Conceptualization, H.H., Y.L., H.-Q.Q., and P.M.A.S.; methodology, Y.L., H.-Q.Q., X.C., J.Q.; formal analysis, Y.L., H.-Q.Q., J.Q.; investigation, Y.L., H.-Q.Q., X.C.; resources, H.H., L.T., J.G., and P.M.A.S.; data curation, Y.L., H.-Q.Q., X.C., J.Q.; writing-Y.L., H.-Q.Q.; writingreview and editing, H.H., and P.M.A.S.; supervision, H.H. All authors have read and agreed to the published version of the manuscript.

Funding: The study and the APC were supported by Institutional Development Funds from the Children's Hospital of Philadelphia to the Center for Applied Genomics, The Children's Hospital of Philadelphia Endowed Chair in Genomic Research to HH.

Institutional Review Board Statement: The protocol was approved by the Institutional Review Boards of Children's Hospital of Philadelphia.

Informed Consent Statement: Not applicable.

Data Availability Statement: The data presented in this study are available in the Supplementary Materials.

Acknowledgments: All authors have read the journal's authorship agreement and that the manuscript has been reviewed by and approved by all named authors. DLPFC data were generated as part of the CommonMind Consortium supported by funding from Takeda Pharmaceuticals Company Limited, F. Hoffman-La Roche Ltd., and from NIH grants R01MH085542, R01MH093725, P50MH066392, P50MH080405, R01MH097276, RO1-MH-075916, P50M096891, P50MH084053S1, R37MH057881, and 
R37MH057881S1, HHSN271201300031C, AG02219, AG05138, and MH06692. Brain tissue for the study was obtained from the following brain bank collections: the Mount Sinai NIH Brain and Tissue Repository, the University of Pennsylvania Alzheimer's Disease Core Center, the University of Pittsburgh NeuroBioBank and Brain and Tissue Repositories, and the NIMH Human Brain Collection Core. CMC Leadership: Pamela Sklar, Joseph Buxbaum (Icahn School of Medicine at Mount Sinai), Bernie Devlin, David Lewis (University of Pittsburgh), Raquel Gur, Chang-Gyu Hahn (University of Pennsylvania), Keisuke Hirai, Hiroyoshi Toyoshiba (Takeda Pharmaceuticals Company Limited), Enrico Domenici, Laurent Essioux (F. Hoffman-La Roche Ltd.), Lara Mangravite, Mette Peters (Sage Bionetworks), Thomas Lehner, and Barbara Lipska (NIMH).

Conflicts of Interest: The authors declare no conflict of interest.

\section{References}

1. Sleiman, P.; Hakonarson, H. From schizophrenia risk locus to schizophrenia genes. Nat. Med. 2016, 22, 583-584. [CrossRef]

2. Chang, X.; Lima, L.D.A.; Liu, Y.; Li, J.; Li, Q.; Sleiman, P.M.A.; Hakonarson, H. Common and Rare Genetic Risk Factors Converge in Protein Interaction Networks Underlying Schizophrenia. Front. Genet. 2018, 9, 434. [CrossRef] [PubMed]

3. Vawter, M.P.; Barrett, T.; Cheadle, C.; Sokolov, B.P.; Wood III, W.H.; Donovan, D.M.; Webster, M.; Freed, W.J.; Becker, K.G. Application of cDNA microarrays to examine gene expression differences in schizophrenia. Brain Res. Bull. 2001, 55, 641-650. [CrossRef]

4. Katsel, P.; Davis, K.; Gorman, J.; Haroutunian, V. Variations in differential gene expression patterns across multiple brain regions in schizophrenia. Schizophr. Res. 2005, 77, 241-252. [CrossRef] [PubMed]

5. Glaus, P.; Honkela, A.; Rattray, M. Identifying differentially expressed transcripts from RNA-seq data with biological variation. Bioinformatics 2012, 28, 1721-1728. [CrossRef] [PubMed]

6. Honkela, A.; Girardot, C.; Gustafson, E.H.; Liu, Y.-H.; Furlong, E.E.; Lawrence, N.D.; Rattray, M. Model-based method for transcription factor target identification with limited data. Proc. Natl. Acad. Sci. USA 2010, 107, 7793-7798. [CrossRef]

7. Liu, Y.; Chang, X.; Hahn, C.-G.; Gur, R.E.; Sleiman, P.A.M.; Hakonarson, H. Non-coding RNA dysregulation in the amygdala region of schizophrenia patients contributes to the pathogenesis of the disease. Transl. Psychiatry 2018, 8, 1-10. [CrossRef]

8. Tian, T.; Wei, Z.; Chang, X.; Liu, Y.; Gur, R.E.; Sleiman, P.M.; Hakonarson, H. The Long Noncoding RNA Landscape in Amygdala Tissues from Schizophrenia Patients. EBioMedicine 2018, 34, 171-181. [CrossRef]

9. Emerelo, V.; Edurand, D.; Lescallette, A.R.; Vrana, K.E.; Hong, L.E.; Faghihi, M.A.; Ebellon, A. Associating schizophrenia, long non-coding RNAs and neurostructural dynamics. Front. Mol. Neurosci. 2015, 8, 57. [CrossRef]

10. Gibbons, A.; Udawela, M.; Dean, B. Non-Coding RNA as Novel Players in the Pathophysiology of Schizophrenia. Non-Coding RNA 2018, 4, 11. [CrossRef]

11. Hoffman, G.E.; Bendl, J.; Voloudakis, G.; Montgomery, K.S.; Sloofman, L.; Wang, Y.-C.; Shah, H.R.; Hauberg, M.E.; Johnson, J.S.; Girdhar, K.; et al. CommonMind Consortium provides transcriptomic and epigenomic data for Schizophrenia and Bipolar Disorder. Sci. Data 2019, 6, 1-14. [CrossRef]

12. Wang, J.; Duncan, D.; Shi, Z.; Zhang, B. WEB-based GEne SeT AnaLysis Toolkit (WebGestalt): Update 2013. Nucleic Acids Res. 2013, 41, W77-W83. [CrossRef]

13. Croft, D.; O’Kelly, G.; Wu, G.; Haw, R.; Gillespie, M.; Matthews, L.; Caudy, M.; Garapati, P.; Gopinath, G.; Jassal, B.; et al. Reactome: A database of reactions, pathways and biological processes. Nucleic Acids Res. 2010, 39, D691-D697. [CrossRef]

14. Mi, H.; Muruganujan, A.; Ebert, D.; Huang, X.; Thomas, P.D. PANTHER version 14: More genomes, a new PANTHER GO-slim and improvements in enrichment analysis tools. Nucleic Acids Res. 2019, 47, D419-D426. [CrossRef]

15. Fromer, M.; Roussos, P.; Sieberts, S.K.; Johnson, J.S.; Kavanagh, D.H.; Perumal, T.M.; Ruderfer, D.M.; Oh, E.C.; Topol, A.; Shah, H.R.; et al. Gene expression elucidates functional impact of polygenic risk for schizophrenia. Nat. Neurosci. 2016, 19, 1442-1453. [CrossRef]

16. Schizophrenia Working Group of the Psychiatric Genomics Consortium. Biological insights from 108 schizophrenia-associated genetic loci. Nat. Cell Biol. 2014, 511, 421-427. [CrossRef]

17. Pardiñas, A.F.; Holmans, P.; Pocklington, A.J.; Escott-Price, V.; Ripke, S.; Carrera, N.; Legge, S.E.; Bishop, S.; Cameron, D.; Hamshere, M.L.; et al. Common schizophrenia alleles are enriched in mutation-intolerant genes and in regions under strong background selection. Nat. Genet. 2018, 50, 381-389. [CrossRef] [PubMed]

18. Fernandes, J.C.R.; Acuña, S.M.; Aoki, J.I.; Floeter-Winter, L.M.; Muxel, S.M. Long Non-Coding RNAs in the Regulation of Gene Expression: Physiology and Disease. Non-Coding RNA 2019, 5, 17. [CrossRef] [PubMed]

19. Goff, L.A.; Groff, A.F.; Sauvageau, M.; Trayes-Gibson, Z.; Sanchez-Gomez, D.B.; Morse, M.; Martin, R.D.; Elcavage, L.E.; Liapis, S.C.; Gonzalez-Celeiro, M.; et al. Spatiotemporal expression and transcriptional perturbations by long noncoding RNAs in the mouse brain. Proc. Natl. Acad. Sci. USA 2015, 112, 6855-6862. [CrossRef] [PubMed]

20. Joung, J.; Engreitz, J.M.; Konermann, S.; Abudayyeh, O.O.; Verdine, V.K.; Aguet, F.; Gootenberg, J.S.; Sanjana, N.E.; Wright, J.B.; Fulco, C.P.; et al. Genome-scale activation screen identifies a lncRNA locus regulating a gene neighbourhood. Nat. Cell Biol. 2017, 548, 343-346. [CrossRef] [PubMed] 
21. Huang, D.W.; Sherman, B.T.; Lempicki, R.A. Bioinformatics enrichment tools: Paths toward the comprehensive functional analysis of large gene lists. Nucleic Acids Res. 2009, 37, 1-13. [CrossRef]

22. Kong, Y.; Yu, T. A Deep Neural Network Model using Random Forest to Extract Feature Representation for Gene Expression Data Classification. Sci. Rep. 2018, 8, 1-9. [CrossRef] [PubMed]

23. Van Ijzendoorn, D.G.P.; Szuhai, K.; Bruijn, I.H.B.-D.; Kostine, M.; Kuijjer, M.L.; Bovée, J.V.M.G. Machine learning analysis of gene expression data reveals novel diagnostic and prognostic biomarkers and identifies therapeutic targets for soft tissue sarcomas. PLoS Comput. Biol. 2019, 15, e1006826. [CrossRef] [PubMed]

24. Hung, T.; Chang, H.Y. Long noncoding RNA in genome regulation: Prospects and mechanisms. RNA Biol. 2010, 7, 582-585. [CrossRef] [PubMed]

25. Gejman, P.V.; Sanders, A.R.; Duan, J. The Role of Genetics in the Etiology of Schizophrenia. Psychiatr. Clin. N. Am. 2010, 33, 35-66. [CrossRef]

26. Alachkar, A.; Wang, L.; Yoshimura, R.; Hamzeh, A.R.; Wang, Z.; Sanathara, N.; Lee, S.M.; Xu, X.; Abbott, G.W.; Civelli, O. Prenatal one-carbon metabolism dysregulation programs schizophrenia-like deficits. Mol. Psychiatry 2017, 23, 282-294. [CrossRef] [PubMed]

27. Smith, P.D.; Coulson-Thomas, V.J.; Foscarin, S.; Kwok, J.C.; Fawcett, J.W. "GAG-ing with the neuron": The role of glycosaminoglycan patterning in the central nervous system. Exp. Neurol. 2015, 274, 100-114. [CrossRef]

28. Emaeda, N. Proteoglycans and neuronal migration in the cerebral cortex during development and disease. Front. Neurosci. 2015, 9, 98. [CrossRef]

29. Berretta, S.; Pantazopoulos, H.; Markota, M.; Brown, C.; Batzianouli, E.T. Losing the sugar coating: Potential impact of perineuronal net abnormalities on interneurons in schizophrenia. Schizophr. Res. 2015, 167, 18-27. [CrossRef] [PubMed]

30. Hoosain, F.G.; Choonara, Y.E.; Tomar, L.K.; Kumar, P.; Tyagi, C.; du Toit, L.C.; Pillay, V. Bypassing P-Glycoprotein Drug Efflux Mechanisms: Possible Applications in Pharmacoresistant Schizophrenia Therapy. BioMed Res. Int. 2015, 2015, 1-21. [CrossRef]

31. Berridge, M.J. Dysregulation of neural calcium signaling in Alzheimer disease, bipolar disorder and schizophrenia. Prion 2013, 7, 2-13. [CrossRef] [PubMed]

32. Stepan, J.; Anderzhanova, E.; Gassen, N.C. Hippo Signaling: Emerging Pathway in Stress-Related Psychiatric Disorders? Front. Psychiatry 2018, 9, 715. [CrossRef]

33. Dobin, A.; Davis, C.A.; Schlesinger, F.; Drenkow, J.; Zaleski, C.; Jha, S.; Batut, P.; Chaisson, M.; Gingeras, T.R. STAR: Ultrafast universal RNA-seq aligner. Bioinformatics 2013, 29, 15-21. [CrossRef]

34. Harrow, J.; Frankish, A.; Gonzalez, J.M.; Tapanari, E.; Diekhans, M.; Kokocinski, F.; Aken, B.L.; Barrell, D.; Zadissa, A.; Searle, S.; et al. GENCODE: The reference human genome annotation for The ENCODE Project. Genome Res. 2012, 22, 1760-1774. [CrossRef] [PubMed]

35. Trapnell, C.; Williams, B.A.; Pertea, G.; Mortazavi, A.; Kwan, G.; Van Baren, M.J.; Salzberg, S.L.; Wold, B.J.; Pachter, L. Transcript assembly and quantification by RNA-Seq reveals unannotated transcripts and isoform switching during cell differentiation. Nat. Biotechnol. 2010, 28, 511-515. [CrossRef] [PubMed]

36. Pedregosa, F.; Varoquaux, G.; Gramfort, A.; Michel, V.; Thirion, B.; Grisel, O.; Blondel, M.; Prettenhofer, P.; Weiss, R.; Dubourg, V.; et al. Scikit-learn: Machine learning in Python. J. Mach. Learn. Res. 2011, 12, 2825-2830.

37. Lin, Y.; Ghazanfar, S.; Wang, K.Y.X.; Gagnon-Bartsch, J.A.; Lo, K.K.; Su, X.; Han, Z.-G.; Ormerod, J.T.; Speed, T.P.; Yang, P.; et al. scMerge leverages factor analysis, stable expression, and pseudoreplication to merge multiple single-cell RNA-seq datasets. Proc. Natl. Acad. Sci. USA 2019, 116, 9775-9784. [CrossRef]

38. Iacob, E.; Light, A.R.; Donaldson, G.W.; Okifuji, A.; Hughen, R.W.; White, A.T.; Light, K.C. Gene Expression Factor Analysis to Differentiate Pathways Linked to Fibromyalgia, Chronic Fatigue Syndrome, and Depression in a Diverse Patient Sample. Arthritis Rheum. 2015, 68, 132-140. [CrossRef] [PubMed]

39. Wu, Y.; Yao, Y.-G.; Luo, X.-J. SZDB: A Database for Schizophrenia Genetic Research. Schizophr. Bull. 2016, 43, 459-471. [CrossRef] [PubMed] 